\title{
Production and properties of the native Chromobacterium violaceum fucose-binding lectin (CV-IIL) compared to homologous lectins of Pseudomonas aeruginosa (PA-IIL) and Ralstonia solanacearum (RS-IIL)
}

Correspondence

Nechama Gilboa-Garber garben@mail.biu.ac.il

Received 7 September 2005 Revised 10 November 2005 Accepted 11 November 2005

\author{
Keren Zinger-Yosovich, ${ }^{1}$ Dvora Sudakevitz, ${ }^{1}$ Anne Imberty, ${ }^{2}$ \\ Nachman C. Garber ${ }^{1}$ and Nechama Gilboa-Garber ${ }^{1}$ \\ ${ }^{1}$ Faculty of Life Sciences, Bar-Ilan University, Ramat-Gan 52900, Israel \\ ${ }^{2}$ CERMAV-CNRS (Joseph Fourier University), BP 53, 38041 Grenoble, Cedex 09, France
}

\section{INTRODUCTION}

Chromobacterium violaceum is a versatile Gram-negative $\beta$ proteobacterium (Dewhirst et al., 1989) that produces the violet non-diffusible antibiotic pigment violacein (Lichstein \& Van de Sand, 1945; Antonio \& Creczynski-Pasa, 2004) and additional antibiotics and enzymes affecting viruses, bacterial and eukaryotic cells (Duran \& Menck, 2001; Melo et al., 2003; Chernin et al., 1998). This bacterium is a

Abbreviations: AHL, N-acylhomoserine lactone; CV-IIL, Chromobacterium violaceum lectin; PA-IL, Pseudomonas aeruginosa first (galactose-binding) lectin; PA-IIL, $P$ aeruginosa second (fucose $>$ mannose-binding) lectin; OS, quorum sensing; RS-IIL, Ra/stonia solanacearum second (mannose $>$ fucose-binding) lectin. common inhabitant of soil and water confined to tropical and subtropical regions. Generally, it behaves as a saprophyte, but sporadically it becomes an aggressive opportunistic animal (including human) pathogen, causing serious infections with a high mortality in immunodeficient individuals (Pemberton et al., 1991; Shao et al., 2002; Alves de Brito et al., 2004). In that behaviour, C. violaceum resembles the green pigment (pyocyanin)-producing bacterium Pseudomonas aeruginosa, which is a worldwide inhabitant of similar ecosystems and has become an important common nosocomial pathogen, endangering hospitalized chronic patients. Apart from their clinical and pharmacological significance, these two bacteria contribute to environmental bioremediation (Faramarzi et al., 2004). 
Since the production of violacein is quorum sensing (QS)driven, it has become an important tool for bacterial QS signal bioassays, especially for the $\mathrm{N}$-acylhomoserine lactone autoinducers (AHLs). A C. violaceum mini-Tn5 mutant, CV026 (dependent on exogenous AHL for violacein production), is used as an indicator organism (McClean et al., 1997; Shaw et al., 1997). Such assays are important because AHLs that belong to the class of furanone derivatives (Uroz et al., 2003; Martinelli et al., 2004) are involved in cell-to-cell signalling in Gram-negative bacteria (Fugua et al., 1994; Swift et al., 1999), leading to production of antibiotics, hydrolytic exoenzymes, virulence factors and lectins (Gilboa-Garber et al., 2000; Winzer et al., 2000), all of which contribute to their pathogenicity.

Although the unveiling of the complete genome sequence of C. violaceum revealed its remarkable and exploitable adaptability (Brazilian National Genome Project Consortium, 2003), the strategies underlying its adaptability to human infections have not yet been deeply investigated at the molecular and genetic levels (Uroz et al., 2003).

Comparison of the ORFs of C. violaceum with those of other organisms revealed closest similarity, $17 \cdot 4 \%$, to ORFs of the soil-borne phytopathogen Ralstonia solanacearum (Salanoubat et al., 2002) and $9 \cdot 61 \%$ similarity to ORFs of the soil-borne animal (including human) pathogen $P$. aeruginosa (Stover et al., 2000). One of the C. violaceum homologous ORFs was found to resemble the ORFs of two lectins: $P$. aeruginosa PA-IIL (fucose $>$ mannose; lecB) (Gilboa-Garber et al., 2000) and R. solanacearum RS-IIL (mannose > fucose) (Sudakevitz et al., 2004).

P. aeruginosa produces PA-IIL (11.73 kDa) (Gilboa-Garber, 1982; Garber et al., 1987) in addition to a galactophilic lectin, PA-IL (12.76 kDa) (Gilboa-Garber, 1972). Both lectins are composed of four identical subunits (Mitchell et al., 2002; Ciocci et al., 2003; Imberty et al., 2004). Their production is controlled together with the bacterium's virulence factors (Gilboa-Garber, 1997) by QS and by RpoS (Winzer et al., 2000), and putative luxI-box type elements (transcriptional regulator-binding sites, which are sensitive to the combined stimulatory effects of AHLs and regulator proteins involved in the control of bioluminescence in Vibrio fischeri) were identified upstream of their promoter regions. Our suggestion long ago that PA-IL and PA-IIL contribute to the adhesion and biofilm formation of $P$. aeruginosa (Gilboa-Garber, 1997), enabling its pathogenicity (Gilboa-Garber \& Garber, 1989), was recently confirmed (Diggle et al., 2002; Tielker et al., 2005).

R. solanacearum possesses a PA-IIL-like lectin (RS-IIL), but does not produce a PA-IL-like one (Gilboa-Garber et al., 2000; Sudakevitz et al., 2004). RS-IIL, which is shorter than PA-IIL by one amino acid $(11.60 \mathrm{kDa})$, resembles it in its composition, $\mathrm{Ca}^{2+}$ requirement for activity and a very high sugar affinity (Garber et al., 1987; Sudakevitz et al., 2004). However, it differs from PA-IIL in showing highest affinity to mannose, accompanied by a lower avidity for fucose and related sugars (Sudakevitz et al., 2004).

The ORF of the PA-IIL- and RS-IIL-like putative $C$. violaceum lectin (named CV-IIL) was used for production of a recombinant protein that was shown to display a preferential fucose-binding activity (Wimmerova et al., 2005; M. Wimmerova \& A. Imberty, unpublished) and a native fucose-binding lectin was found in C. violaceum cell extracts (K. Zinger-Yosovich \& N. Gilboa-Garber, unpublished). No PA-IL-like ORF was found in the C. violaceum genome, nor PA-IL-like activity in its cell extracts.

In the present study, native CV-IIL production was examined using both wild-type and the CV026 mutant. The properties of the purified native CV-IIL, isolated from the wild-type, and its interactions with human and animal erythrocytes and with various monosaccharides and EDTA were compared to those of PA-IIL and RS-IIL.

\section{METHODS}

Bacterial strains and cultures. C. violaceum wild-type ATCC 12472, and R. solanacearum ATCC 11696 were purchased from the American Type Culture Collection (ATCC) (Manassas, VA, USA). $P$. aeruginosa ATCC 33347 is our isolate deposited at ATCC and C. violaceum CV026 violacein-negative mini-Tn5 mutant of ATCC 31532 was kindly provided by Professor P. Williams, University of Nottingham, UK.

All the bacteria were grown at $28^{\circ} \mathrm{C}$ with vigorous shaking for 3 days in nutrient broth and supplied with $0 \cdot 4 \%$ trehalose. The harvested cells were washed three times and disintegrated by sonication.

Purified lectin preparations. The preparation of the bacterial (wild-type) cell extracts and the procedures for purification of the three lectins, CV-IIL, PA-IIL and RS-IIL, including heating to $65^{\circ} \mathrm{C}$ for $15 \mathrm{~min}$, ammonium sulfate precipitation (70\% saturation), and affinity chromatography using Sepharose-mannose, were performed as earlier described (Gilboa-Garber, 1982; Sudakevitz et al., 2004). The lectins were eluted from the column by $0 \cdot 1 \mathrm{M}$ EDTA, followed by an overnight dialysis against saline containing $0.01 \mathrm{M} \mathrm{CaCl}_{2}$, followed by another similar dialysis against either PBS (for the haemagglutination tests) or twice-distilled water (for other purposes and for concentration of the preparations). The purified lectin preparations were analysed by SDS-PAGE with Coomassie brilliant blue staining, as previously described (Sudakevitz et al., 2004).

$\mathbf{N}$-terminal amino acid sequencing and mass spectrometry analysis. The purified C. violaceum lectin preparation, giving a single band in SDS-PAGE, was subjected to N-terminal amino acid sequence determination by Edman degradation and to mass spectrometry by the Technion Protein Research Center, as previously described (Sudakevitz et al., 2004).

Analyses of the upstream extragenic sequences of the CVIIL ORF. Homology searching of the upstream (200 bp fragment) sequences of the genes (beginning from their transcription starts) of CV-IIL (locus name CV 1744, http://www.brgene.lncc.br/cviolaceum) and RS-IIL (locus name RSc 3288), was performed as described before for PA-IL and PA-IIL (Gilboa-Garber et al., 2000).

Haemagglutination and tests for its inhibition. Diverse human and animal erythrocytes (kindly supplied by the Magen David Adom National Blood Services in Israel and the animal centre of 
Bar-Ilan University, Israel) were washed three times with phosphatebuffered $(0 \cdot 025 \mathrm{M}, \mathrm{pH} 7 \cdot 2)$ isotonic $\mathrm{NaCl}$ solution (PBS) and then treated by $0 \cdot 1 \%$ papain with $0.01 \%$ cysteine, as previously described (Gilboa-Garber, 1982; Sudakevitz et al., 2004). A $50 \mu \mathrm{l}$ sample of each lectin preparation examined was serially diluted with $50 \mu \mathrm{l}$ PBS to produce twofold dilutions. PBS and erythrocyte suspensions $(50 \mu \mathrm{l}$ each) were added to each tube. After $30 \mathrm{~min}$ at room temperature, the tubes were centrifuged for $30 \mathrm{~s}(1000 \mathrm{~g})$, and the haemagglutinating activity was examined as previously described (Gilboa-Garber, 1982). The activity was represented by the number of tubes in which there was haemagglutination, along the twofold dilutions (e.g. 7 positive tubes $=$ positive reaction up to dilution of $1: 128$, or original activity of 128 haemagglutination units).

In the haemagglutination inhibition test, each solution examined (carbohydrates at $0.3 \mathrm{M}$ or EDTA at $0.1 \mathrm{M}$ ) was serially twofold diluted in $50 \mu \mathrm{l}$ PBS and then $50 \mu$ of the lectin solution (using the highest dilution leading to agglutination of all the erythrocytes in one large mass) was added to each tube. After $30 \mathrm{~min}$ at room temperature, $50 \mu \mathrm{l}$ of $5 \%$ papain-treated human O blood type erythrocyte suspension was added to each tube (Gilboa-Garber, 1982) and, after another $30 \mathrm{~min}$, the haemagglutination was examined as described above.

The haemagglutination inhibition intensity was represented by the highest number of dilutions $\left(\log _{2}\right.$ dilution $\left.^{-1}\right)$ at which no significant haemagglutination occurred.

Statistical evaluation. The results of the haemagglutination and haemagglutination inhibition tests were analysed by Student's $t$-test.

\section{RESULTS}

\section{Haemagglutinating activities of intact $\boldsymbol{C}$. violaceum cells and their extracts and purification of CV-IIL from the extracts}

Examination of intact $C$. violaceum cell suspensions revealed considerable haemagglutinating activities of the cell-surface adhesins. Those included strong sugar- and EDTAinsensitive and -sensitive activities. The crude extracts of the CV026 mutant exhibited much lower haemagglutinating activities as compared to those of the wild-type. Since the fucose/mannose- and EDTA-sensitive activity, resembling that of PA-IIL, was the goal of our research, we purified it from the wild-type $C$. violaceum crude cell extracts, as we did for PA-IIL and RS-IIL, using the procedures described in Methods. In all steps of the purification procedure, the CV-IIL activity behaved like those of PA-IIL and RS-IIL.

Examination of the purified lectin by SDS-PAGE stained with Coomassie brilliant blue (Fig. 1a) revealed the desired single band of CV-IIL with a molecular mass around $11 \cdot 9 \mathrm{kDa}$, close to that of PA-IIL.

\section{$\mathrm{N}$-terminal amino acid sequence of the purified native CV-IIL}

Analysis of the N-terminal amino acid sequence of the purified lectin revealed two molecule populations. Both of them showed exactly the anticipated sequence, AQQGVFTLPARINFGVTVLV, but while the majority of molecules did not bear the initiating methionine (113 amino acids), a minority did contain that methionine (114 amino acids).

\section{Mass spectrometric analysis}

The above two (major and minor) subunit populations were also observed in the mass spectrometric analysis (Fig. 1b). The molecular mass values registered were: $11852 \cdot 6104 \mathrm{Da}$ (without initiating methionine) and 11978.4834 (with that methionine) Da. This and the above results confirmed that the isolated protein was indeed CV-IIL.

\section{Search for a luxl-box-like sequence in CV-IIL and RS-IIL gene promoters}

Since CV-IIL and RS-IIL, like PA-IIL, were found to be produced in the late stationary phase of the bacteria (3 days), we looked for luxI-box-like sequences upstream of their genes using the luxI-box and luxI-box-type sequence of PA-IIL (located 24-43 bp upstream of its ORF). That alignment revealed partial sequence similarity 101-120 and 115-134 bp upstream of the transcriptional starts of the CV-IIL and RS-IIL genes, respectively (Fig. 2). (a)

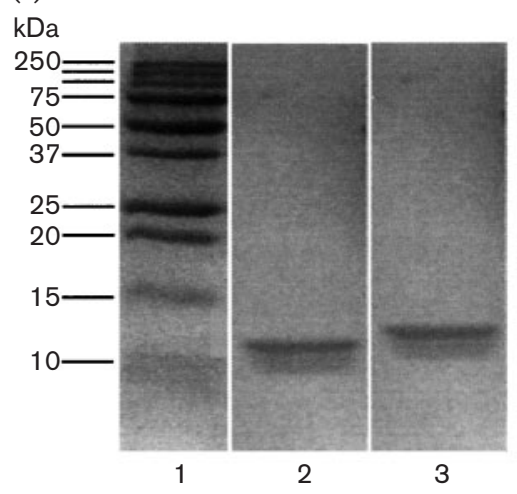

(b)

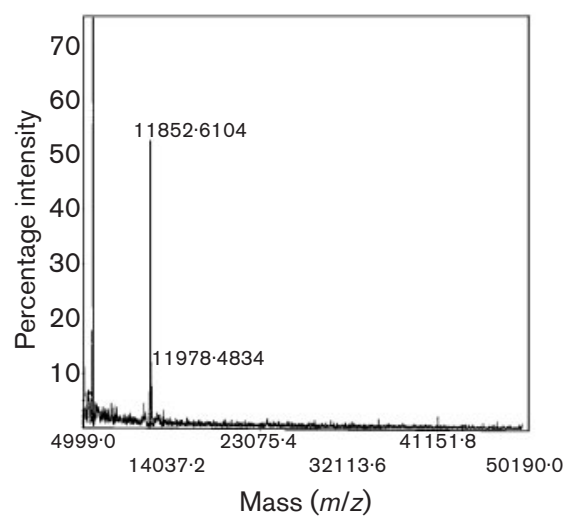

Fig. 1. (a) SDS-PAGE of purified CV-IIL (3) and PA-IIL (2), stained by Coomassie brilliant blue; (b) mass spectroscopic analysis of CV-IIL. 


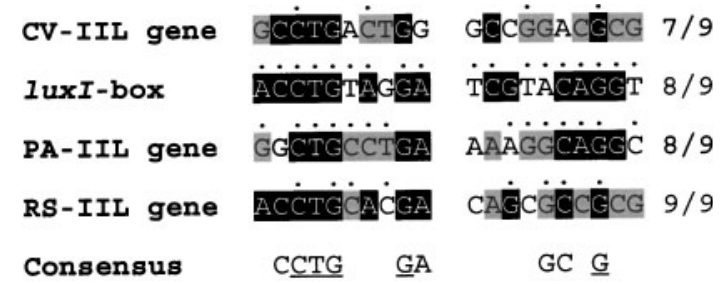

Fig. 2. luxl-box-like $20 \mathrm{bp}$ nucleotide sequences (possible AHL-binding sites) upstream of the genes encoding CV-IIL (locus name CV 1744), PA-IIL (Gilboa-Garber et al., 2000) and RS-IIL (locus name RSc 3288). The points represent complementary symmetry between the first and second $10 \mathrm{bp}$ segments composing the $20 \mathrm{bp}$ of each box. Identity with luxl-box basepairs is indicated by black, and similarity between two or three of the four by grey. The consensus $9 \mathrm{bp}$ include those representing $4 / 4$ boxes (underlined) and those representing $3 / 4$ boxes. The numbers on the right represent the basepairs fitting the consensus $9 \mathrm{bp}$.

\section{Comparison of the haemagglutinating activities of CV-IIL, PA-IIL and RS-IIL towards human erythrocytes of different $A B O$ types}

Using adult human $\mathrm{H}$-positive red blood cells of types $\mathrm{O}, \mathrm{A}$, $B$ and $A B$, it was found that the three lectins agglutinated all of them regardless of their $\mathrm{ABO}$ types (Fig. 3). However, when $\mathrm{O}_{\mathrm{h}}$ Bombay-type erythrocytes, lacking the $\alpha 1-2$ fucosyl residue, were examined, CV-IIL, like PA-IIL, agglutinated them very weakly; RS-IIL did interact with those H-negative erythrocytes, although at somewhat reduced intensity. Fetal erythrocytes obtained from cord bloods were agglutinated very well by all three lectins.

\section{Comparison of the haemaggluatinating activities of CV-IIL, PA-IIL and RS-IIL towards different animal erythrocytes}

Using human (O-type), cow, sheep, rabbit and rat erythrocytes, it became apparent that there are some differences not only between CV-IIL (Fuc > Man) and RS-IIL (Man > Fuc), but also between CV-IIL and PA-IIL, which is closer to it in preferential fucose affinity. As seen in Fig. 4, the three lectins did not react at all with cow erythrocytes while reacting well with human, sheep and rat erythrocytes. However, whereas PA-IIL strongly agglutinated the rabbit, rat and sheep erythrocytes, and RS-IIL most strongly agglutinated the sheep cells followed by the rabbit red blood cells, CV-IIL most strongly agglutinated the rat erythrocytes and did not agglutinate the rabbit cells at all.

\section{Comparison of the monosaccharide specificity and sensitivity to EDTA of CV-IIL and PA-IIL}

Comparison of the inhibition the haemagglutinating activities of purified CV-IIL and PA-IIL by EDTA and a series of monosaccharides (Fig. 5) revealed that these two lectins, which do not interact with D-glucose or D-galactose, are very

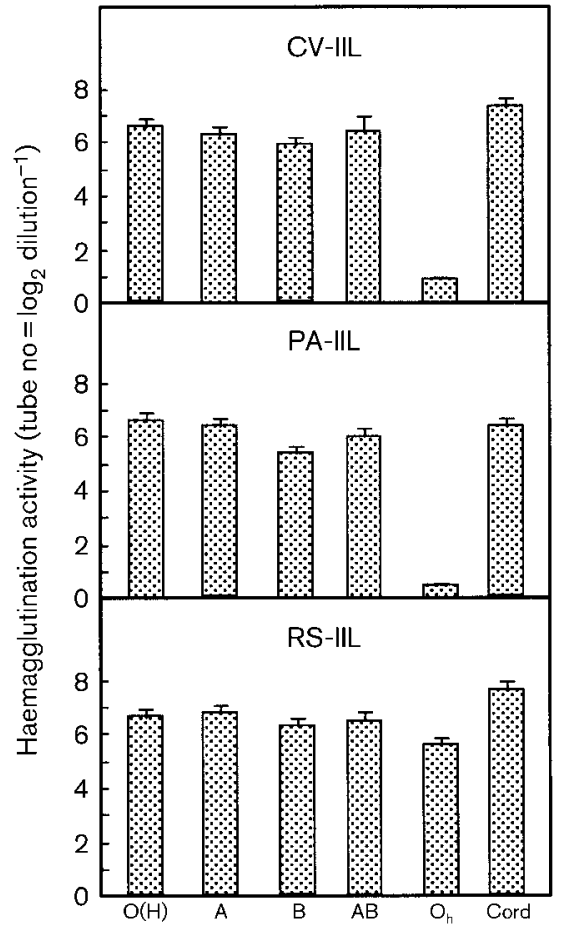

Fig. 3. Comparison of the haemagglutinating activities of CV-IIL, PA-IIL and RS-IIL towards papain-treated human erythrocytes of different $A B O$ types from adults and from fetal (cord) bloods. The data represent means of at least six experiments \pm SEM.

similar in their EDTA sensitivity and Fuc $>$ Ara $>$ Fru $>$ Man specificity order (with subtle affinity differences). An exception to the order similarity is the higher affinity of CV-IIL to L-galactose than to L-fucose, not observed with PA-IIL or RS-IIL.

\section{DISCUSSION}

In the last decade the availability of the whole-genome sequences of the closely related opportunistic pathogenic bacteria P. aeruginosa (Stover et al., 2000), R. solanacearum (Salanoubat et al., 2002) and C. violaceum (Brazilian National Genome Project Consortium, 2003) has enabled comparative studies of the molecular basis, properties and function of their virulence factors and regulation of their production. The central pillars of the virulence arsenals of these bacteria are lectins, which, by virtue of their sugarbinding, contribute to the pathogens' host specificity and adherence to host cells, facilitating the establishment of infection. The first lectins of these bacteria, PA-IL and PAIIL, were discovered in P. aeruginosa cell extracts (GilboaGarber, 1972; Gilboa-Garber 1982) before the genome sequence of this bacterium had been determined. Their gene sequences and regulation of their production have since been studied (Gilboa-Garber, 1997; Gilboa-Garber et al., 2000; Winzer et al., 2000), as well as their 3D structures and their interactions with saccharides (Mitchell et al., 2002; 


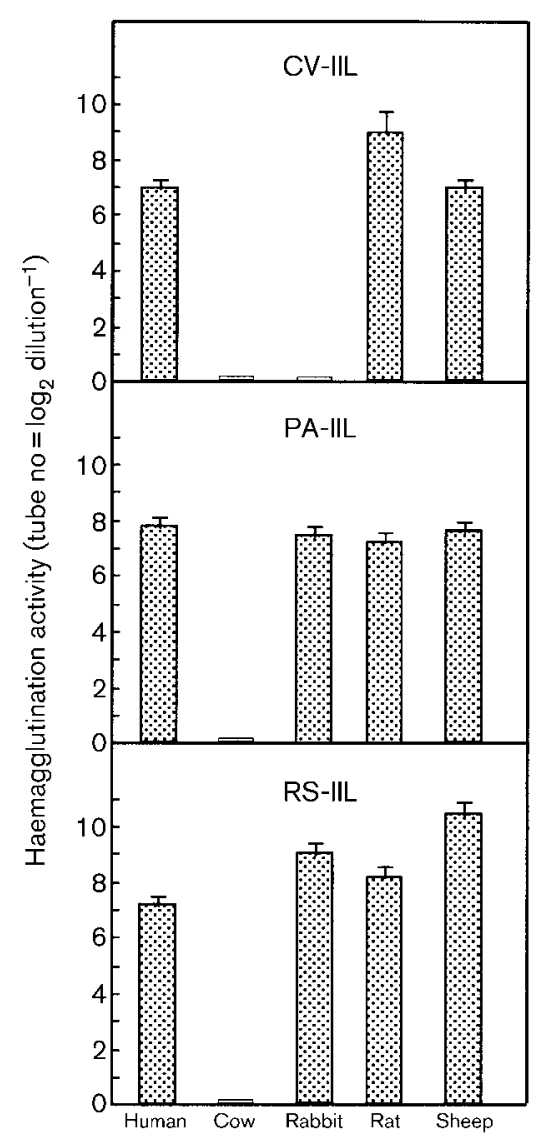

Fig. 4. Comparison of the haemagglutinating activities of CVIIL, PA-IIL and RS-IIL towards papain-treated human (O-type) and diverse animal erythrocytes. The data represent means of at least six experiments \pm SEM.

Ciocci et al., 2003; Imberty et al., 2004) for insight into the molecular basis of their host glycan recognition. The existence of RS-IIL, which is homologous to PA-IIL, was deduced following an alignment study comparing the PA-IIL sequence with the newly published $R$. solanacearum genome sequence (Sudakevitz et al., 2004). Recombinant RS-IIL was produced soon after, and both the native and recombinant lectins were analysed. The crystal 3D structure of the lectin interacting with the saccharides for which it has high affinity showed that minute differences in amino acid composition between RS-IIL and PA-IIL determine the variation in their sugar affinities - the preferential affinity of PA-IIL for L-fucose versus that of RS-IIL for D-mannose (Sudakevitz et al., 2004).

With the release of the $C$. violaceum genome sequence, a third homologous lectin was deduced based on alignment search using PA-IIL against the $C$. violaceum genome. The alignment information indicated a homologous ORF of the same size as PA-IIL and RS-IIL, differing from them only in a few amino acids (Sudakevitz et al., 2004). The putative lectin ORF was cloned in E. coli and the recombinant CV-IIL protein was found to be a tetrameric lectin which

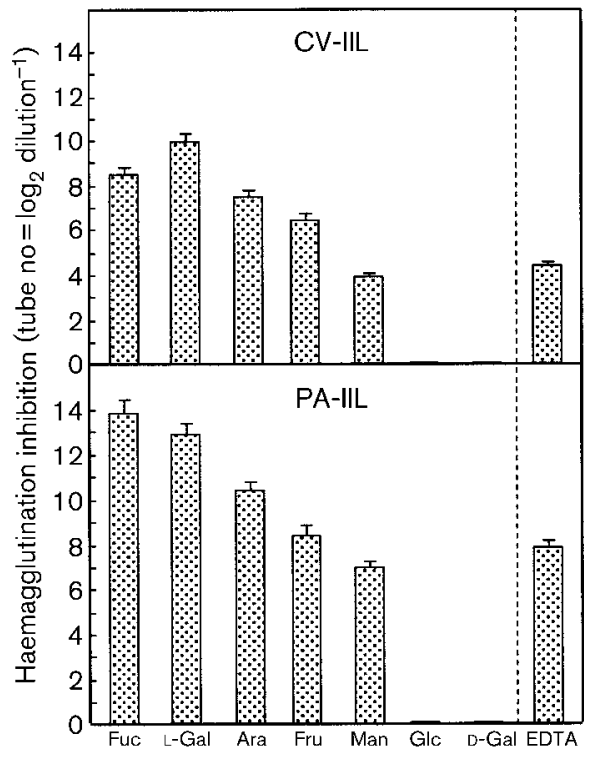

Fig. 5. Comparison of the inhibition of CV-IIL and PA-IIL by decreasing concentrations of various monosaccharides (beginning at $0.3 \mathrm{M}$ ) and EDTA (beginning at $0.1 \mathrm{M}$ ). The data represent means of at least six experiments \pm SEM.

resembles PA-IIL in L-fucose preference; its crystal 3D structures in interactions with fucose and mannose derivatives are currently being investigated (M. Wimmerova \& A. Imberty, unpublished).

The present study showed that the purified native $C$. violaceum lectin is similar to PA-IIL in its molecular mass (around $11.9 \mathrm{kDa}$ ). Two molecule populations (with and without the initiating methionine) are clearly observed. The $\mathrm{N}$-terminal amino acid analysis data fit the predicted sequence and confirm the native CV-IIL homology to PAIIL and RS-IIL and to the recombinant lectin of Wimmerova et al. (2005).

As for the regulation of CV-IIL and RS-IIL formation: (a) these two lectins, like native PA-IIL, are mainly produced (preferentially under osmotically balanced conditions) in the late stationary phase; (b) the C. violaceum mutant, CV026, which displays repressed autoinducer formation, produces very low lectin activity under the same growth conditions; and (c) like PA-IL and PA-IIL, which are known to be under QS cascade control (Gilboa-Garber et al., 2000; Winzer et al., 2000) their gene upstream regions contain luxI-box-like 20 bp sequences (Fig. 2) supposed to bind QS signals for their gene expression. Taken together these data may be regarded as an indirect indication that the production of both CV-IIL and RS-IIL, like those of PAIL and PA-IIL, might be driven by QS; however, this proposal has to be proven experimentally.

The results of the haemagglutination tests (Figs 3 and 4) show that while CV-IIL resembles PA-IIL (more than 
RS-IIL) in selective agglutination of H-positive erythrocytes, it differs from PA-IIL (and RS-IIL) in showing no interaction with rabbit cells.

In accordance with results obtained with the recombinant CV-IIL (Wimmerová et al., 2005; M. Wimmerova \& A. Imberty, personal communication), the native lectin exhibits EDTA sensitivity and a very high L-fucose affinity, followed by somewhat lower arabinose, fructose and Dmannose avidities, and does not react at all with either Dglucose or D-galactose (Fig. 5). In its higher fucose versus mannose affinity, CV-IIL is similar to PA-IIL but not to RSIIL. However, it differs from PA-IIL, and also from RS-IIL, in L-galactose $>\mathrm{L}$-fucose affinity, which is probably related to small amino acid variations that are associated with stronger attraction of the CV-IIL binding site by the 6th $\mathrm{CH}_{2} \mathrm{OH}$ of L-galactose while the other two lectins are more strongly attracted by the terminal $\mathrm{CH}_{3}$ of L-fucose. The amino acid motif 22-24, which is involved in monosaccharide specificity, is Ser-Ser-Gly in PA-IIL, Ala-Ala-Asn in RS-IIL and Ser-Ala-Ala in CV-IIL (Imberty et al., 2004; Sudakevitz et al., 2004).

\section{Conclusions}

Native CV-IIL $(11.9 \mathrm{kDa})$ is homologous to PA-IIL $(11 \cdot 7)$ and RS-IIL (11.6) in its structure and properties, including sugar and EDTA sensitivity, and in the regulation of its production. It is also similar to those lectins in its interactions with $\mathrm{H}$-positive human erythrocytes regardless of their $\mathrm{ABO}$ type, and with rat and sheep erythrocytes, but not cow erythrocytes. In its selectivity to $\mathrm{H}$-positive cells and stronger fucose versus mannose affinity, CV-IIL is closer to PA-IIL than to RS-IIL. This similarity might be related to their common host spectrum, which is mainly animal while that of RS-IIL is mainly plant. However, it differs from both PA-IIL and RS-IIL in showing no interaction with rabbit erythrocytes and in its L-galactose $>$ L-fucose preference.

\section{ACKNOWLEDGEMENTS}

The authors thank Dr Shai Uliel for help with the alignment of the $R$. solanacearum and C. violaceum upstream nucleotide sequences, and Ms Sharon Victor, Ms Avrille Goldreich and Ms Ela Gindy for their help in the preparation of this manuscript. This research is part of K. Z.Y.'s PhD thesis.

\section{REFERENCES}

Alves de Brito, C. F., Carvalho, C. M. B., Santos, F. R., Gazzinelli, R. T., Oliveira, S. C., Azevedo, V. \& Teixeira, S. M. R. (2004). Chromobacterium violaceum genome: molecular mechanisms associated with pathogenicity. Genet Mol Res 3, 148-161.

Antonio, R. V. \& Creczynski-Pasa, T. B. (2004). Genetic analysis of violacein biosynthesis by Chromobacterium violaceum. Genet Mol Res 3, 85-91.

Brazilian National Genome Project Consortium. (2003). The complete genome sequence of Chromobacterium violaceum reveals remarkable and exploitable bacterial adaptability. Proc Natl Acad Sci U S A 100, 11660-11665.

Chernin, L. S., Winson, M. K., Thompson, J. M., Haran, S., Bycroft, B. W., Chet, I., Williams, P. \& Stewart, G. S. (1998). Chitinolytic activity in Chromobacterium violaceum: substrate analysis and regulation by quorum sensing. J Bacteriol 180, 4435-4441.

Ciocci, G., Mitchell, E. P., Cautier, C., Wimmerova, M., Sudakevitz, D., Perez, S., Gilboa-Garber, N. \& Imberty, A. (2003). Structural basis of calcium and galactose recognition by the lectin PA-IL of Pseudomonas aeruginosa. FEBS Lett 555, 297-301.

Dewhirst, F. E., Paster, B. J. \& Bright, P. L. (1989). Chromobacterium, Eikenella, Kingella, Neisseria, Simonsiella, and Vitreoscilla species comprise a major branch of the beta group Proteobacteria by $16 \mathrm{~S}$ ribosomal ribonucleic acid sequence comparison: transfer of Eikenella and Simonsiella to the family Neisseriaceae (emend.). Int J Syst Bacteriol 39, 258-266.

Diggle, S. P., Winzer, K., Lazdunski, A., Williams, P. \& Camara, M. (2002). Advancing the quorum in Pseudomonas aeruginosa: MvaT and the regulation of $\mathrm{N}$-acylhomoserine lactone production and virulence gene expression. J Bacteriol 184, 2576-2586.

Duran, N. \& Menck, C. F. (2001). Chromobacterium violaceum: a review of pharmacological and industrial perspectives. Crit Rev Microbiol 27, 201-222.

Faramarzi, M. A., Stagars, M., Pensini, E., Krebs, W. \& Brandl, H. (2004). Metal solubilization from metal-containing solid materials by cyanogenic Chromobacterium violaceum. J Biotechnol 113, 321-326.

Fugua, W. C., Winans, S. C. \& Greenberg, E. P. (1994). Quorum sensing in bacteria: the LuxR-LuxI family of cell density-responsive transcriptional regulators. J Bacteriol 176, 269-275.

Garber, N., Guempel, U., Gilboa-Garber, N. \& Doyle, R. J. (1987). Specificity of the fucose-binding lectin of Pseudomonas aeruginosa. FEMS Microbiol Lett 48, 331-334.

Gilboa-Garber, N. (1972). Inhibition of broad spectrum hemagglutinin from Pseudomonas aeruginosa by D-galactose and its derivatives. FEBS Lett 20, 242-244.

Gilboa-Garber, N. (1982). Pseudomonas aeruginosa lectins. Methods Enzymol 83, 378-385.

Gilboa-Garber, N. (1997). Multiple aspects of Pseudomonas aeruginosa lectins. Nova Acta Leopold 75, 153-177.

Gilboa-Garber, N. \& Garber, N. (1989). Microbial lectin cofunction with lytic activities as a model for a general basic lectin role. FEMS Microbiol Rev 63, 211-222.

Gilboa-Garber, N., Katcoff, D. J. \& Garber, N. C. (2000). Identification and characterization of Pseudomonas aeruginosa PA-IIL lectin gene and protein compared to PA-IL. FEMS Immunol Med Microbiol 29, 53-57.

Imberty, A., Wimmerova, M., Mitchell, E. P. \& Gilboa-Garber, N. (2004). Structures of the lectins from Pseudomonas aeruginosa: insights into the molecular basis for host glycan recognition. Microb Infect 6, 221-228.

Lichstein, H. C. \& Van de Sand, V. F. (1945). Violacein, an antibiotic pigment produced by Chromobacterium violaceum. J Infect Dis 76, $47-51$.

Martinelli, D., Grossmann, G., Sequin, U., Brandl, H. \& Bachofen, R. (2004). Effects of natural and chemically synthesized furanones on quorum sensing in Chromobacterium violaceum. BMC Microbiol 4, 25.

McClean, K. H., Winson, M. K., Fish, L. \& 9 other authors (1997). Quorum sensing and Chromobacterium violaceum: exploitation of violacein production and inhibition for the detection of $\mathrm{N}$ acylhomoserine lactones. Microbiology 143, 3703-3711. 
Melo, P. S., Justo, G. Z., de Azevedo, M. B. M., Duran, N. \& Haun, M. (2003). Violacein and its beta-cyclodextrin complexes induce apoptosis and differentiation in HL60 cells. Toxicology 186, 217-225.

Mitchell, E., Houles, C., Sudakevitz, D., Wimmerova, M., Gautier, C., Perez, S., Wu, A. M., Gilboa-Garber, N. \& Imberty, A. (2002). Structural basis for oligosaccharide-mediated adhesion of Pseudomonas aeruginosa in the lung of cystic fibrosis patients. Nat Struct Biol 9, 918-921.

Pemberton, J. M., Vincent, K. M. \& Penfold, R. J. (1991). Cloning and heterologous expression of the violacein biosynthesis gene cluster from Chromobacterium violaceum. Curr Microbiol 22, 355-358.

Salanoubat, M., Genin, S., Artiguenave, F. \& 25 other authors (2002). Genome sequence of the plant pathogen Ralstonia solanacearum. Nature 415, 497-502.

Shao, P. L., Hsueh, P. R., Chang, Y. C., Lu, C. Y., Lee, P. Y., Lee, C. Y. \& Huang, L. M. (2002). Chromobacterium violaceum infection in children: a case of fatal septicemia with nasopharyngeal abscess and literature review. Pediatr Infect Dis J 21, 707-709.

Shaw, P. D., Ping, G., Daly, S. L., Cha, C., Cronan, J. E., Jr, Rinehart, K. L. \& Farand, S. K. (1997). Detecting and characterizing $N$ acyl-homoserine lactone signal molecules by thin layer chromatography. Proc Natl Acad Sci U S A 94, 6036-6041.

Stover, C. K., Pham, X. Q., Erwin, A. L. \& 28 other authors (2000). Complete genome sequence of Pseudomonas aeruginosa PAO1, an opportunistic pathogen. Nature 406, 959-964.

Sudakevitz, D., Kostlanova, N., Blatman-Jan, G., Mitchell, E. P., Lerrer, B., Wimmerova, M., Katcoff, D. J., Imberty, A. \& Gilboa-Garber, N.
(2004). A new Ralstonia solanacearum high-affinity mannose-binding lectin RS-IIL structurally resembling the Pseudomonas aeruginosa fucose-specific lectin PA-IIL. Mol Microbiol 52, 691-700.

Swift, S., Williams, P. \& Stewart, G. S. A. B. (1999). Nacylhomoserine lactones and quorum sensing in proteobacteria. In Cell-Cell Signaling in Bacteria, pp. 291-313. Edited by G. M. Dunny \& S. C. Winans. Washington, DC: American Society for Microbiology.

Tielker, D., Hacker, S., Loris, R., Strathmann, M., Wingender, J., Wilhelm, S., Rosenau, F. \& Jaeger, K. E. (2005). Pseudomonas aeruginosa lectin LecB is located in the outer membrane and is involved in biofilm formation. Microbiology 151, 1313-1323.

Uroz, S., D’Angelo-Picard, C., Carlier, A., Elasri, M., Sicot, C., Petit, A., Oger, P., Faure, D. \& Dessaux, Y. (2003). Novel bacteria degrading $N$-acylhomoserine lactones and their use as quenchers of quorum-sensing-regulated functions of plant-pathogenic bacteria. Microbiology 149, 1981-1989.

Wimmerova, M., Mitchell, E. P., Budova, M., Sabin, C., Kostlanova, N., Perret, S., Cioci, G., Gilboa-Garber, N. \& Imberty, A. (2005). PA-II-like lectins: new family of high affinity present in opportunistic bacteria. In Glycoproteomics: Protein Modifications for Versatile Functions, Dubrovnik, 2005, p. 94 (abstract no. 94).

Winzer, K., Falconer, C., Garber, N. C., Diggle, S. P., Camara, M. \& Williams, P. (2000). The Pseudomonas aeruginosa lectins PA-IL and PA-IIL are controlled by quorum sensing and by RpoS. J Bacteriol 182, 6401-6411. 